\title{
Simulation, Modeling and Programming for Autonomous Robots: The Open Source Perspective
}

\author{
Herman Bruyninckx \\ Katholieke Universiteit Leuven \\ Department of Mechanical Engineering \\ Celestijnenlaan 300B \\ B3001 Leuven \\ Belgium \\ http://people.mech.kuleuven. be/ bruyninc \\ http://www.mech.kuleuven.be/robotics/acm/
}

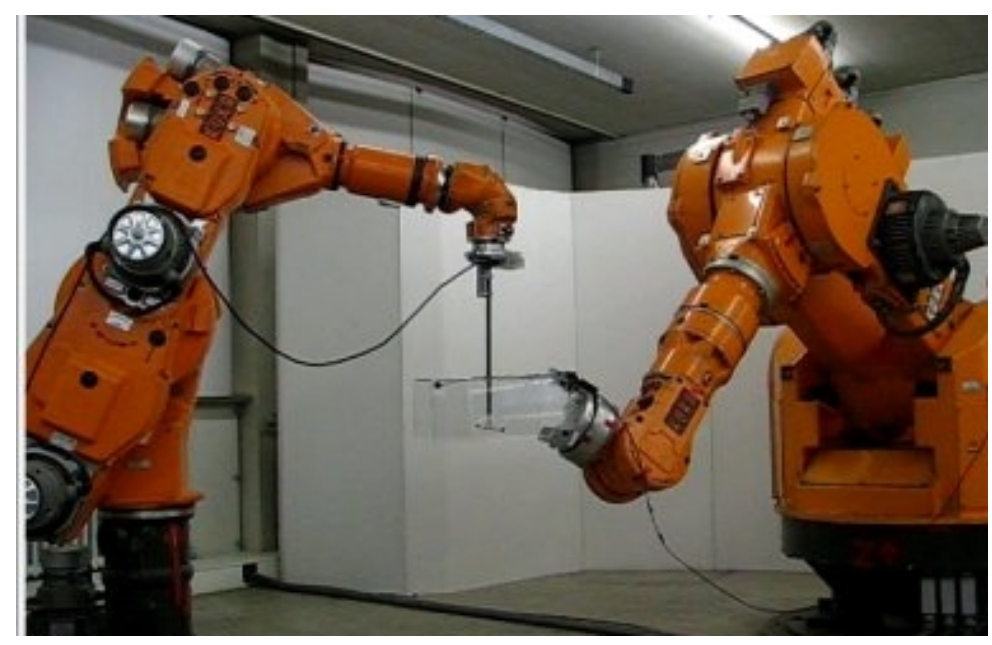

Dr. Bruyninckx has been active in open source robot control software development since the year 2000, and has created the Orocos project that targets (realtime) simulation and control of complex robot systems. In this talk, he will present a broad vision on which software components exist, or have still to be developed, in open source, in order to reach an all-encompasing, powerful and vertically integrated software stack that supports all possible aspects of advanced robotics research and development. The talk gives an overview of current and future projects that work towards these goals, and of the difficult problem of having these projects work towards a common set of long-term objectives. The presentation also indentifies several practical, technical, legal and commercial hurdles, to be taken by participants (both academic and industrial) that are part of the current open source ecosystems, or that are interested in becoming part of such ecosystem. 